\title{
On the transition from 3D to 2D transport equations for a study of long-term cosmic-ray intensity variations in the heliosphere
}

\author{
M.S. Kalinin, ${ }^{a}$ B.B. Gvozdevsky, ${ }^{b}$ M.B. Krainev, ${ }^{a, *}$ O.P.M. Aslam, ${ }^{c}$ M.D. Ngobeni ${ }^{c, d}$ \\ and M.S. Potgieter ${ }^{e}$ \\ ${ }^{a}$ Lebedev Physical Institute, Russian Academy of Sciences, 119991 Moscow, Russia \\ ${ }^{b}$ Polar Geophysical Institute, Russian Academy of Sciences, 184209 Apatity, Russia \\ ${ }^{c}$ Centre for Space Research, North-West University, 2520 Potchefstroom, South Africa \\ ${ }^{d}$ School of Physical and Chemical Sciences, North-West University, 2745 Mmabatho, South Africa \\ ${ }^{e}$ Institute for Experimental and Applied Physics, CA University in Kiel, 24118 Kiel, Germany \\ E-mail: kalinin273@sci.lebedev.ru, gvozdevsky@pgia.ru, mkrainev46@mail.ru, \\ aslamklr2003@gmail.com, Donald.Ngobeni@nwu.ac.za, \\ Marius.S.Potgieter@gmail.com
}

The exact two-dimensional (2D) transport equation (TPE) for galactic cosmic ray (GCR) intensity in the heliosphere averaged over longitude, derived by averaging the full three-dimensional (3D) steady-state TPE over longitude is considered. This exact 2D TPE is equal to that with the averaged 3D TPE coefficients but with the "source-term" $Q$ due to 3D modulation effects. In particular, $Q$ is equal to the longitude convolution of the longitudinal variances of the coefficients and the intensity as used in the 3D TPE and as applicable to the modulation of GCR intensity. In previous work we suggested an expression for $Q$ without solving the 3D TPE for the simplest case when the only characteristic heliospheric feature depending on helio-longitude is the polarity of the heliospheric magnetic field.

The motivation for the estimating and studying the structure of the source-term of the exact 2D TPE is two-fold: 1) solving 2D instead of 3D TPE for simulating the long-term variations of GCR intensity looks more easy and logical and 2) studying the structure of the source-term can improve our understanding of the simulated GCR modulation.

This study is focused on calculating and analizing the same longitude convolution as $Q$ numerically, solving the steady-state 3D TPE for the above mentioned simplest case. Beside studying the structure of this calculated source-term, $Q 1$, it is also compared with that estimated without solving the 3D TPE, $Q 2$.

$37^{\text {th }}$ International Cosmic Ray Conference (ICRC 2021)

July 12 th - 23rd, 2021

Online - Berlin, Germany

\footnotetext{
${ }^{*}$ Presenter
} 


\section{Introduction}

Presently it is practice for the description of the long-term modulation of the GCR intensity to solve the three dimensional (3D) transport equation (TPE) for the phase density (or distribution function) $\mathcal{U}(\vec{r}, p)=\mathcal{J}(\vec{r}, T) / p^{2}$, where $p, T$ and $\mathcal{J}$ are the momentum, kinetic energy and intensity of these particles, respectively. The procedure then is to average $\mathcal{U}$ over longitude $\left(U=\langle\mathcal{U}\rangle_{\varphi}\right)$ and the corresponding intensity is compared with observations averaged over a solar rotation period $(\approx 27$ days) [14]. Probably, it would be easier to use for this purpose the 2D TPE for the phase density averaged over longitude. However, when we use for such an equation the exact analogue of the 3D TPE but with all coefficients averaged over longitude, the results of the two approaches are sometimes rather different, which requires further investigation.

Previously, in [6], an internally justified procedure was suggested for the reduction of the full 3D TPE to the 2D TPE for the longitudinal averaged phase density. This version of the 2D TPE differs from what was discussed in the previous paragraph by a 'source' term, for which exact calculation we need to know the solution of the full 3D TPE. For quite some time we have tried to estimate this 'source' term by assuming approximations and at last seem to have succeeded for the simplest case when the only longitude dependent heliospheric factor is the heliospheric magnetic field (HMF) polarity (see [7] and references therein). However, there are still some remaining questions about the procedure used and the generality of this estimation.

The most direct way to study the structure of this term, and to check the procedure of its estimation, is to calculate it from the outcome of a full 3D TPE using an up-to-date and comprehensive steady-state 3D code $[1,10,13,16]$, which is the purpose of this paper. In section 2 the procedure for the 3D to 2D TPE reduction is discussed and followed by the calculation of both 3D and 2D drift velocities in section 3. In section 4 the source term in the 2D TPE is calculated and compared with both the drift term of the same equation and the estimated value of the source term.

\section{Reduction of 3D to 2D TPE}

The distribution of the GCR phase density $\mathcal{U}$ in the heliosphere with respect to momentum $p$ is described (see [11], [8], [12]) by the 3D TPE in the steady-state approximation in a coordinate system rotating with the Sun:

$$
\nabla(\mathcal{K} \nabla \mathcal{U})-\overrightarrow{\mathcal{V}}^{s w *} \cdot \nabla \mathcal{U}-\overrightarrow{\mathcal{V}}^{d r} \cdot \nabla \mathcal{U}+\frac{\mathcal{D} \mathcal{V}}{3} p \frac{\partial \mathcal{U}}{\partial p}=0
$$

where $\mathcal{K}$ is the diffusion tensor, $\overrightarrow{\mathcal{V}}^{s w *}=\overrightarrow{\mathcal{V}}^{s w}-\vec{\Omega} \times \vec{r}$, and $\vec{\Omega}, \overrightarrow{\mathcal{V}}^{d r}, \overrightarrow{\mathcal{V}}^{s w}, \mathcal{D} \mathcal{V}$ are the angular rotation velocity of the Sun, particle drift and solar wind velocities and the divergence of the latter, respectively. This TPE is set in the inner part of the domain and the usual boundary conditions are specified. As previously done [6], we split the boundary problem for $\mathcal{U}=U+u$ into a set of two equations for $U$ and $u$ by decomposing the coefficients of (1) into longitudinally averaged ones and the longitude variances, $\mathcal{K}=K+k, \overrightarrow{\mathcal{V}}^{s w}=\vec{V}^{s w}+\vec{v}^{s w}, \overrightarrow{\mathcal{V}}^{d r}=\vec{V}^{d r}+\vec{v}^{d r}, \mathcal{D V}=D V+d v$, then averaging (1) over longitude, and obtaining:

$$
\nabla(K \nabla U)-\vec{V}^{s w} \cdot \nabla U-\vec{V}^{d r} \cdot \nabla U+\frac{D V}{3} p \frac{\partial U}{\partial p}+Q=0
$$


The source term $Q=-\left\langle\vec{v}^{d r} \cdot \nabla u\right\rangle_{\varphi}$ in (2) takes into account that the only longitude dependent heliospheric factor in our model is the HMF polarity, so $k, \vec{v}^{s w}, d v$ are all equal to zero and the only non zero variance of the TPE coefficients is that of the drift velocity.

Subtracting (2) from (1), one gets the 3D TPE for the variance of the phase density, $u$, and it is this 3D TPE for $u$, which was studied in our previous efforts to estimate the source term in (2) without solving (1).

\section{The 3D and 2D drift velocities}

According to the above mentioned models there is no longitudinal dependence in the solar wind velocity so that its divergence, the strength and geometry of the HMF, the diffusion coefficients and TPE coefficients as well as the proton non modulated spectrum (taken as the very local interstellar spectrum) are not be discussed again here. However, the drift velocity which is the only non-axialsymmetric TPE coefficient, and its structure and how to proceed with its longitudinal averaging, are described in some detail.

The particle drift velocity can be expressed as:

$$
\overrightarrow{\mathcal{V}}^{d r}=C_{\text {scat }}^{d r} \cdot \frac{p v}{3 q}\left[\nabla \times\left(\overrightarrow{\mathcal{B}} / \mathcal{B}^{2}\right)\right]
$$

where $C_{s c a t}^{d r}$ is a function of rigidity describing drift reduction caused by particle scattering, e.g. [10]; with $v$ and $q$ the velocity and charge of particles, and $\overrightarrow{\mathcal{B}}$ and $\mathcal{B}$ the HMF vector and its magnitude (strength). All the entities in (3) specified in front of the vector product do not depend on longitude.

The regular HMF can be represented as $\overrightarrow{\mathcal{B}}=\mathcal{F} \overrightarrow{\mathcal{B}}^{m}$, where $\mathcal{F}$ is the HMF polarity, i.e., a scalar function positive in the positive sectors and negative in negative sectors, changing sign across the heliospheric current sheet (HCS) surface, $\mathcal{F}(r, \vartheta, \varphi)=0$. By $\overrightarrow{\mathcal{B}}^{m}$ we designated the unipolar (or "monopolar") magnetic field (with unit vector $\vec{b}_{m}$ ) equal to $\overrightarrow{\mathcal{B}}$ in the positive sectors and having the reversed polarity in the negative ones. In our case this monopolar HMF does not depend on longitude and it is a modification of Parker's HMF according to [15] as implemented by [16].

The first approximation of the HMF polarity can be expressed as $\mathcal{F}(x)=A[2 H(x)-1]$, with $A$ and $H(x)$ being the polarity (the sign of the radial component of the HMF in the high-latitude $\mathrm{N}$-hemisphere) and the Heaviside function of the distance $x$ from the HCS, respectively. The value of $x$ is positive in the $\mathrm{N}$-hemisphere. Then $\nabla \mathcal{F}=2 A \delta(x) \overrightarrow{\mathbf{n}}_{c s}$, where $\overrightarrow{\mathbf{n}}_{c S}$ is the unit vector normal to the HCS surface and directed to the N-hemisphere and $\overrightarrow{\mathbf{e}}_{d r}=\left[\overrightarrow{\mathbf{n}}_{c s} \times \vec{b}_{m}\right]$ is the unit vector in the direction of the HCS drift in the monopolar HMF. However, in the model and code the more sophisticated model for $\mathcal{F}$ is used which allows the smooth transition of polarity between the negative and positive sectors with the depth of the HCS about four Larmor radii, $r_{L}$, see [3-5] and references therein.

Next, decomposing the drift velocity into regular $\overrightarrow{\mathcal{V}}^{d r, \text { reg }}$ and HCS $\overrightarrow{\mathcal{V}}^{d r, c s}$ parts with rather 
different space features, gives:

$$
\begin{aligned}
\overrightarrow{\mathcal{V}}^{d r, \text { reg }} & =C_{\text {scat }}^{d r} \cdot \frac{p v}{3 q} \cdot \mathcal{F}\left[\nabla \times \frac{\vec{B}^{m}}{B^{2}}\right], \\
\overrightarrow{\mathcal{V}}^{d r, c s} & =C_{\text {scat }}^{d r} \cdot \frac{p v}{3 q} \cdot\left[\nabla \mathcal{F} \times \frac{\vec{B}^{m}}{B^{2}}\right] .
\end{aligned}
$$

These expressions are valid for any HCS surface. In case of the usually used tilted HCS (as the great circle tilted with respect to the solar equator with a so-called tilt angle $\alpha_{t}$ at any $r$ ) the HCS surface is specified as

$$
\vartheta^{c s}=\frac{\pi}{2}-\arctan \left(\tan \alpha_{t} \sin \left(\varphi+\frac{\Omega\left(r-r_{s s}\right)}{V^{s w}}\right)\right),
$$

where $r_{s s}$ is the radius of the source surface of the HMF.

When averaged over longitude, the HMF polarity and the drift velocities are:

$$
\begin{aligned}
F & =\langle\mathcal{F}\rangle_{\varphi} \\
\vec{V}^{d r, \text { reg }} & =C_{\text {scat }}^{d r} \cdot \frac{p v}{3 q} \cdot F\left[\nabla \times \frac{\vec{B}^{m}}{B^{2}}\right], \\
\vec{V}^{d r, c s} & =C_{\text {scat }}^{d r} \cdot \frac{p v}{3 q} \cdot\left[\langle\nabla \mathcal{F}\rangle_{\varphi} \times \frac{\vec{B}^{m}}{B^{2}}\right] .
\end{aligned}
$$

\section{Source term for the 2D TPE}

The boundary-value problem to be solved is comprised of the 3D TPE (1) and the usual boundary conditions (see e.g., [13]). For the purpose of this paper it is not important which set of parameters of the models for the TPE coefficients is used and we proceed by selecting the parameter set for the second half of 2007, with the HMF polarity indicated as $\mathrm{A}<0$, and when there was a pronounced 27 day variations in the GCR proton intensity [9]. For the time being the best description of the observations around sunspot minimum 23/24 is achieved with the models and parameters listed and described by $[1,2,14,16]$.

Because of space limitations, we illustrate only the structure of the calculated source term $Q 1(r, \theta)$ and compare it with the drift term, $T^{d r}=-\vec{V}^{d r} \cdot \nabla U$, of the 2D TPE (2) and with the estimated source term $Q 2(r, \theta)$ for a fixed kinetic energy of $T=1 \mathrm{GeV}$ and calculated by solving the 3D TPE (1). For this energy, we save the intensity and all drift velocities (all three components of the regular and HCS parts of the velocity) and also the HMF polarity $\mathcal{F}$ in all nodes of the numerical space grid; the numbers of nodes in $r, \theta, \phi$ are 350:181:181. The illustrations of intermediate characteristics important for understanding how the structures of $Q 1, T^{d r}$ and $Q 2$ are formed are collected in the file Kalinin_et_al_ICRC-2021_ID353_Appendix.pdf which is saved as an additonal file. We refer to these figures using the number of the figure in this file with the capital letter A (e.g., Fig. 5A).

Since our main task is to demonstrate the general structure of the source term in the global heliosphere, we use for all figures a 2D formatting and colour presentation of the characteristics of 


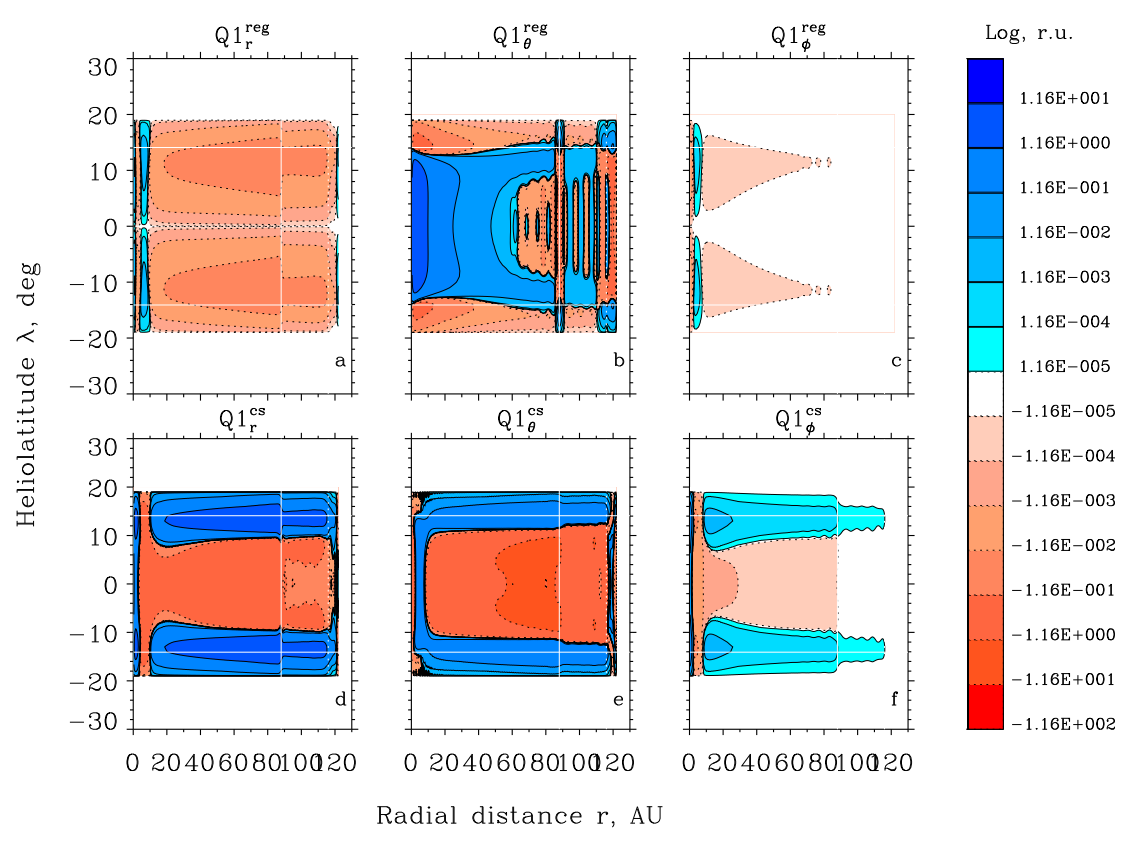

Figure 1: Spatial distribution of the radial (left panels), co-latitude (central panels) and longitude (right panels) parts of the calculated source term $Q 1$ in (2) caused by regular (upper panels) and current sheet drifts (lower panels) shown separately. For the general details of all figures (scale, units, white lines) see the text.

interest according to a log (sometimes linear) scale of shades of colour. It means that the difference between values for successive contours (the boundaries between adjacent shades of color) is one order of magnitude (for log scale) or constant value (for linear scale). So as to not go deeper into the units of different characteristics we always use the relative units (r.u.).

The white lines in all figures both here and in Appendix file show the form of HCS and its latitudinal boundaries (according to (6)) - wavy lines for $f(\phi, \lambda)$-figures, and horizontal lines for $f(r, \lambda)$-figures; the position of the termination shock and a layer near the heliopause (horizontal lines for $f(\phi, r)$-figures and vertical lines for $f(r, \lambda)$-figures, solid for $r_{T S}$ and dotted for layer before the heliopause).

When discussing the main features of these figures, we do not emphasize the details which can be seen in the outer heliosphere (near the termination shock and heliopause) and also in the inner heliosphere, close to the Sun (at $r<10 \mathrm{AU}$ ), although these details being very interesting.

In Fig. 1 the spatial distribution of the radial, colatitude and longitude parts of the calculated source term are shown caused by regular drift (upper panels) and HCS drift (lower panels). Evidently, the source term is non zero only in and around the HMF sector zone, in our case (with $\alpha_{t}=14.6 \mathrm{deg}$ and $T=1 \mathrm{GeV}$ ) for $|\lambda|<20 \mathrm{deg}$.

In Fig. 2 the space distribution of the drift term $T^{d r}=-\vec{V}^{d r} \cdot \nabla U$ of 2D TPE is shown in the same format and also in the same latitude range even though the drift term $T^{d r}$ is non zero in the higher latitudes as well (see Figs. 27A-29A). The spotty (or fast alternating in sign) structure of the co-latitude part of the drift term caused by drift along the HCS (Fig. 2, d), is probably the result of the calculation with finite grid (that is, finite differences in all three directions) and it means that 


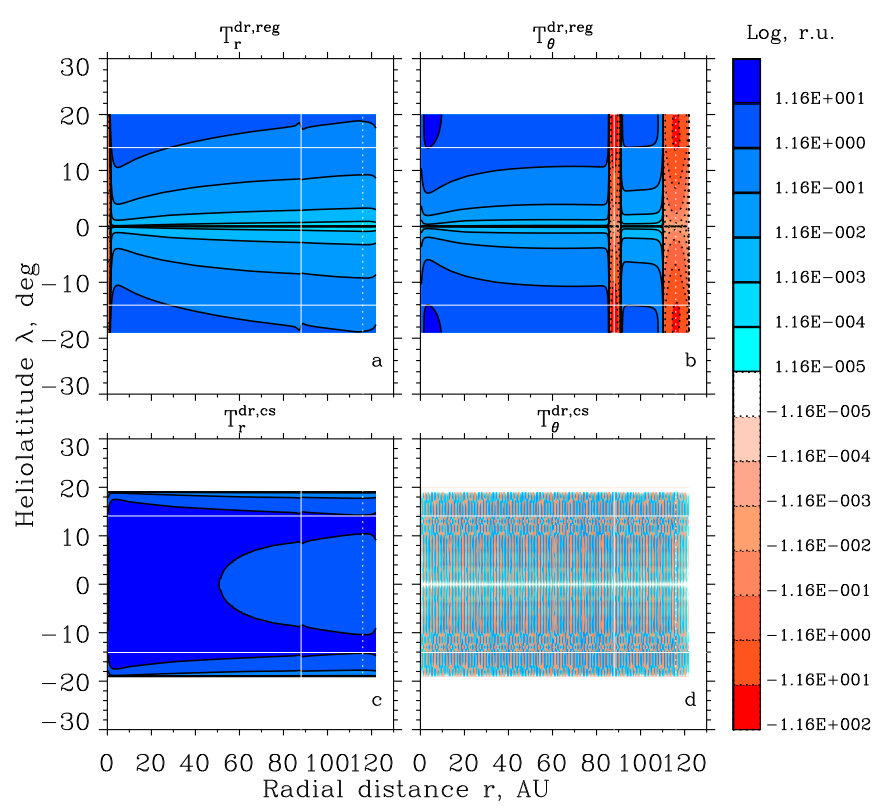

Figure 2: Spatial distribution of the radial and co-latitude parts of the drift term $T^{d r}=-\vec{V}^{d r} \cdot \nabla U$ in (2) caused by regular and current sheet drifts shown separately. For the general details of all figures (scale, units, white lines) see the text.

this part is close to zero.

The first impression one gets from a comparison of the Figs. 1 and 2 is that the signs of the corresponding parts of the $Q 1$ and $T^{d r}$ are opposite which means that the source effectively reduces the corresponding components of drift. The intriguing feature of the calculated $Q 1$ is the strong co-latitude parts due to both the regular (Fig. 1b) and HCS (Fig. 1e) drifts. The formation of the structure of any part of $Q 1$ can be studied from the figures in the appendix mentioned above. For instance, the co-latitude part of $Q 1$ due to the HCS drift $Q 1_{\theta}^{c s}=-\left\langle v_{\theta}^{d r, c s} \cdot \nabla_{\theta} u\right\rangle_{\varphi}$ shown in Fig. 1e is formed from the longitude convolution of the variance of the co-latitude component HCS-drift, $v_{\theta}^{d r, c s}$, shown in Fig. 19A of the Appendix (see also Figs. 10A and 14A), with the co-latitude component of gradient of the longitude variance of intensity, $\nabla_{\theta} u$, shown in Fig. 25A (see also Figs. 21A and 23A).

As mentioned above [7] got the approximate expression for the source term as:

$$
\begin{aligned}
Q 2 & =C_{\text {scat }}^{d r} \cdot \frac{p v}{3 q}\left[\nabla \times \Phi \frac{\overrightarrow{\mathcal{B}}^{2}}{\mathcal{B}}\right] \cdot \nabla U, \\
\Phi(F) & =\left(1-F^{2}\right)^{0.5} \cdot \arcsin F,
\end{aligned}
$$

and its regular and current sheet parts respectively as: 


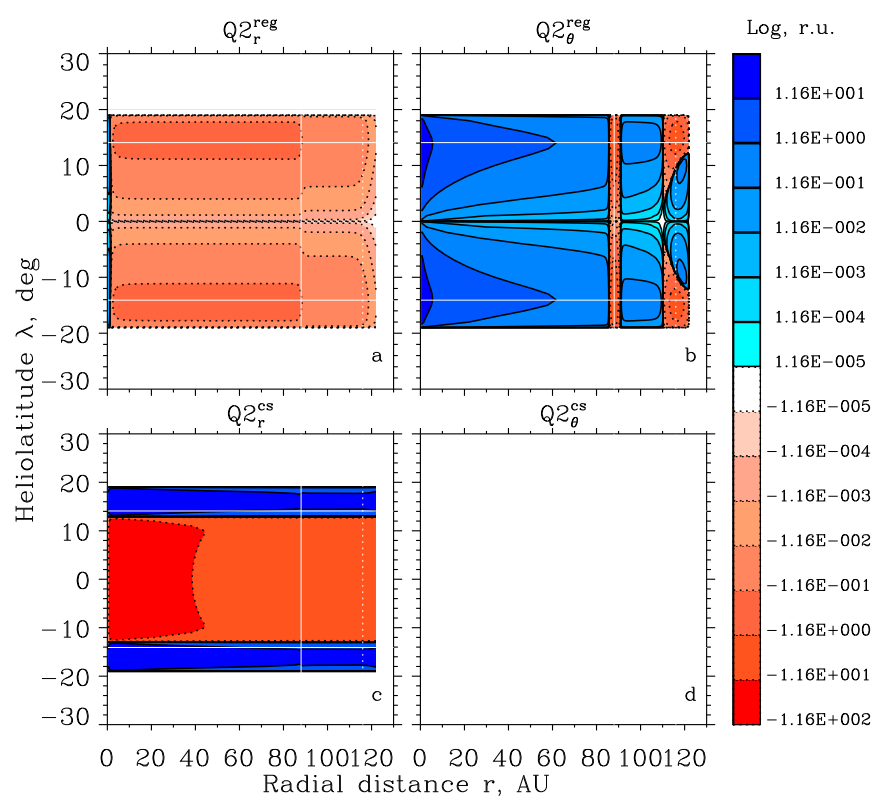

Figure 3: Spatial distribution of the radial and co-latitude parts of the source term $Q 2$ of (2) caused by the regular and current sheet drifts shown separately as estimated without solving (1). The colatitude part of the estimated source term $Q 2$ caused by the current sheet drift (panel d) is zero. For the general details of all figures (scale, units, white lines) see the text.

$$
\begin{aligned}
Q 2^{r e g} & =C_{\text {scat }}^{d r} \cdot \frac{p v}{3 q} \cdot \Phi \cdot\left[\nabla \times \frac{\vec{B}^{m}}{B^{2}}\right] \cdot \nabla U, \\
Q 2^{c s} & =C_{s c a t}^{d r} \cdot \frac{p v}{3 q} \cdot\left[\nabla \Phi \times \frac{\vec{B}^{m}}{B^{2}}\right] \cdot \nabla U,
\end{aligned}
$$

A comparison of the structures of the estimated source term $Q 2$ in Fig. 3 and of the calculated source term $Q 1$ in Fig. 1, shows that: (1) The absence in $Q 2$ of the longitude parts which are present (although rather small) in $Q 1$; (2) The qualitative similarity between the radial parts (although too large for $Q 2_{r}^{c s}$ in Fig. 3 (c)); and much smaller for $Q 2$ the co-latitude parts due to both the regular and HCS drifts.

One possible cause of the descrepancy between $Q 1$ and $Q 2$ could be that in deriving the estimate (10-11) for $Q 2$ in [7] the approximation of infinitely thin HCS was used discussed in section 3. Besides, the unexpected features of the calculated $Q 1$ could be taken into account in our further efforts to improve the estimation of the source term without solving 3D TPE.

\section{Conclusions}

The structure of the calculated source term of the exact 2D TPE reduced from the 3D TPE steady-state in the frame rotating with the Sun for the simple case of the HMF polarity as the only 
longitude dependent heliospheric factor is rather complex and on the whole should result in some reduction of drift effects in the solar modulation of GCRs.

A detailed analysis is needed for more precise conclusions since the contribution of the source term is different for different parts of the drift term (for different components of the drift velocity caused by both the regular and the current sheet drifts) and for different energies.

On the whole, the structure of the source term of the exact 2D TPE, estimated earlier without solving the 3D TPE, differs from its structure calculated using the solutions of the 3D TPE, although there are similar features. Although these calculations assist us in estimating the source term properly, further study and improvement are required.

\section{Acknowledgments}

MBK acknowledges the partial support of RFBR-NRF grant 19-52-60003 SA-t. MDN thanks the South African (SA) NRF for partial financial support under Joint Science and Technology Research Collaboration between SA and Russia (grant no: 118915).

\section{References}

[1] Aslam O.P.M., Bisschoff D. and Potgieter M.S. Proc. Sci. (ICRC2019), 358, 1054 (2019).

[2] Bisschoff D., Potgieter M. S. and Aslam O. P. M., Astrophys. J. 878, 59, 2019.

[3] Burger R., Moraal H. and Webb G.M., Astrophys. Space Sci. 116, 107, 1985.

[4] Burger R.A. and Potgieter M.S., Astrophys. J. 339, 501, 1989.

[5] Hattingh M. and Burger, R.A. Adv. Space Res. 16, 213, 1995.

[6] Kalinin M.S. and Krainev M.B. Proc. 24th ICRC (Rome) 4, 688, 1995.

[7] Kalinin, M.S. and Krainev, M.B., Geomag. \& Aeron., Pleiades Publishing, Ltd. 54, 4, 423 2014.

[8] Krymskiy G. F., Geomag. \& Aeron. 4, 763, 1964.

[9] Modzelewska R., Bazilevskaya G.A., Boezio M. et al., Astrophys. J., 904, 3, 2020.

[10] Ngobeni M.D. and Potgieter M.S., Adv. Space Res. 48, 300, 2015.

[11] Parker E.N., Phys. Rev., 110, 1445, 1958.

[12] Parker, E.N., Planet. Space Sci. 13, 9, 1965.

[13] Potgieter M.S., Living Rev. Solar Phys., 10, 3, 2013.

[14] Potgieter M.S., Vos E.E., Boezio M., et al., Solar Phys. 289, 391, 2014.

[15] Smith C.W. and Bieber J.W., Astrophys. J. 370, 435, 1991

[16] Vos, E.E. and Potgieter. M.S., Astrophys. J. 815, 119, 2015. 